Article

\title{
Critical Lines Identification for Skeleton-Network of Power Systems under Extreme Weather Conditions Based on the Modified VIKOR Method
}

\author{
Chang Han ${ }^{1}$ (D), Yuxuan Zhao ${ }^{1}$, Zhenzhi Lin ${ }^{1, *}$, Yi Ding ${ }^{1}$, Li Yang ${ }^{1}$, Guanqiang Lin ${ }^{2}$, \\ Tianwen $\mathrm{Mo}^{2}$ and Xiaojun $\mathrm{Ye}^{2}$ \\ 1 School of Electrical Engineering, Zhejiang University, Hangzhou 310027, China; hc2399@163.com (C.H.); \\ yuxuanzhao@aliyun.com (Y.Z.); yiding@zju.edu.cn (Y.D.); eeyangli@zju.edu.cn (L.Y.) \\ 2 Electric Power Dispatching and Control Center, Huizhou Power Supply Bureau of Guangdong Power Grid \\ Co., Ltd., Huizhou 516000, China; 13500177552@139.com (G.L.); motw_csg@139.com (T.M.); \\ yeagnes@foxmail.com (X.Y.) \\ * Correspondence: zhenzhi.lin@gmail.com; Tel.: +86-571-87951542
}

Received: 7 May 2018; Accepted: 22 May 2018; Published: 25 May 2018

\begin{abstract}
Identifying and preferentially reinforcing critical lines for skeleton-network of power systems is significant in improving the secure and stable operation of power systems under extreme weather conditions. Under this background, in this paper, six indexes are first presented for identifying critical lines for skeleton-network with the power elements' parameters and the impact of extreme weather conditions, the network topology and the operation state of power systems considered. Then, the modified Vise Kriterijumska Optimizacija I Kompromisno Resenje in Serbian (VIKOR) method, in which the synthetic weights of indexes determined by the combination weighting method are adopted, is utilized to identify the importance degrees of lines in a given power system. Both the overall performance and the outstanding individual performance of lines are considered, which is beneficial for the critical lines identification for skeleton-network. Finally, the proposed multi-indexes and methods are applied to part of the actual Guangdong power system in China. The numerical results are compared with those obtained by single-attribute and multi-attribute evaluation methods and other evaluation methods.
\end{abstract}

Keywords: critical lines identification; skeleton-network; extreme weather; network topology; operation state; the modified VIKOR method

\section{Introduction}

With the rapid development of economy, people require more reliable and secure power supply [1]. However, power systems are probably operated under extreme weather conditions in some areas. Many typhoon disasters happen in China's coastal areas and a complete or partial blackout occurred frequently [2]. For example, typhoon "Rainbow" attacked Guangdong power system in China on 4 October 2015 and caused a partial blackout and huge economic cost. Research shows that the removal of a few critical lines could cause the propagation of failures [3]. In view of this, Guangdong Power Grid Corporation in China proposed an action plan which aims at preventing critical nodes and transmission lines in the identified skeleton-network from damages caused by extreme weather conditions through preventive measures (e.g., power tower reinforcement work). At present, how to select the critical lines of the skeleton-network for resisting extreme weather such as typhoons is an urgent problem to be solved for Guangdong's power system.

Under this background, it is necessary to identify the critical lines for determining the skeleton-network of the power system concerned. Vulnerability identification method, which is based 
on the impact of line removal on a given power system, is utilized to identify the critical lines. Several typical vulnerability identification methods have been proposed, including fault chain theory [4], complex network theory [5], risk theory [6] and entropy theory [7]. These methods could be classified into two types. The first type is to identify the critical lines based on the topologic characteristics. In [8], the rate of change of the average network efficiency before and after the disconnection of power elements is proposed to measure the importance degrees of the power elements. In [9,10], an improved maximum flow based on complex network approach for topology analysis is proposed to identify the critical lines in a power system. The other type is to identify the critical lines based on the operation state of power systems. In [11], hybrid flow betweenness index, which covers the power flow's direction and the line's maximum transmission capacity, is defined to identify critical lines with a comprehensible physical background. In [12], the power flow index and vulnerability index are defined based on geometry of flow path to analyze the impact of lines fault on a given power system, and then to identify the critical lines. In [13], a vulnerable transmission line identification method is proposed using the improved structural hole theory, converting the critical lines identification in power systems into critical nodes evaluation in correlation networks. In [14], an integrated "betweenness" index is utilized to identify critical lines with the line's role in the transmission of power flow and the impact of removing lines on power systems considered. In [15], three indexes (i.e., the weighted reactance betweenness index, the relation degree index based on node degree and the status importance index based on active power) considering both operation state and network topology of power systems are proposed for the critical lines identification. Furthermore, the lines with high failure probabilities, which are easy to fail under extreme weather, should also be considered in reinforcement work for the skeleton-network. In [16], a fuzzy inference system based on fuzzy clustering method and a regional weather model are combined to analyze the impacts of adverse weather on the reliability of power elements.

Thus far, several indexes have been proposed for identifying critical lines, while only one single index is adopted in some existing publications (e.g., [9-11]). It may not be enough to cover the impact of various factors of a complex power system on the identification results of critical lines. Furthermore, those indexes are utilized to evaluate the importance degrees of lines in a power system under normal operation states, and hence cannot be directly utilized to identify critical lines for the skeleton-network. In addition, the lines with high failure probabilities under extreme weather have not been considered by those indexes. Although multi-indexes evaluation method (i.e., utility theory and cooperative game) for critical lines identification is proposed in [15], only the overall performances of lines are considered, which is not suitable for determining the skeleton-network with a diversity of critical lines. Thus, how to extract the comprehensive characteristic parameters of the complex power system and select a reasonable and effective evaluation method to identify the critical lines for the skeleton-network is a key problem that needs to be studied deeply. Given this background, a multi-indexes critical lines identification method is proposed in this paper, and the modified Vise Kriterijumska Optimizacija I Kompromisno Resenje in Serbian (VIKOR) method is utilized to identify the critical lines of a given power system.

The major contributions of this paper are as follows: (1) Six indexes, which consider the power elements' parameter and the impact of extreme weather, and the network topology and operation state of the concerned power system comprehensively, are first presented from different perspectives and synthesized to evaluate the importance degrees of lines for the skeleton-network, especially for critical lines identification under extreme weather conditions such as typhoon. (2) The modified VIKOR method, which takes maximizing outstanding individual performance and group utility as the objective, is first applied to identify the critical lines for the skeleton-network, by which not only the critical lines with large comprehensive importance degrees, but also the critical lines which have great impacts on a certain aspect of power systems can be effectively identified for the skeleton-network effectively. (3) The proposed indexes and methods are applied to an actual power system to determine critical lines for the skeleton-network to resist extreme weather scenarios. 
This paper is organized as follows. Section 2 presents six indexes for line importance degree evaluation. In Section 3, the modified VIKOR method, in which the synthetic weights of indexes determined by the combination weighting method are adopted, is presented to identify the critical lines. In Section 4, numerical results of a part of the actual Guangdong power system in China are presented to illustrate the feature of the proposed multi-indexes line importance degree evaluation method, i.e., critical lines identification method. Finally, conclusions are drawn in Section 5.

\section{Multiple Indexes for Line Importance Degree Evaluation of Complex Power Systems}

According to complex network theory, a real power network can be abstracted as an undirected and weighted graph $G(E, L)$, which consists of a node set $E$ and a line set $L$. Before the critical lines identification, evaluation indexes need to be selected first to evaluate the importance degrees of lines. The main task of skeleton-network optimization is to reserve critical nodes and lines which are important in the topology structure and operation state of a power system. Thus, the line importance degree evaluation indexes should be selected comprehensively. Therefore, with power elements' parameters and the impact of extreme weather, the network topology structure and operation state of the complex power system considered, the following six indexes for identifying the critical lines are presented.

\subsection{Failure Probability of Line under Extreme Weather}

Many transmission lines in the coastal area are vulnerable to typhoons. The vulnerable power facilities within typhoon-affected regions should be paid more attention. In the identification of critical lines, the failure probabilities of the power towers to support transmission lines under typhoon condition should be analyzed first. Generally, the failure probabilities of lines are determined according to the power elements' structure and the wind speed. Thus, it is necessary to build the wind field model of typhoons for determining the failure probability of line.

The Batts model, a wind field model of typhoons, is usually utilized for coastal architecture design in America [17]. The wind speed of a given location could be calculated by the Batts model based on the relationship between the typhoon center and the given location [17]. In the Batts model, the maximum wind radius is defined as

$$
R_{\max }=e^{\left(-0.1239 \Delta P^{0.6003}+5.1034\right)}
$$

The maximum average wind speed at the maximum wind radius $R_{\max }$ is defined as

$$
v_{R_{\max }}=0.865 v_{g x}+0.5 v_{T}
$$

where $v_{T}$ is the typhoon moving speed. $v_{g x}$ is the maximum gradient wind speed and is defined as

$$
v_{g x}=K \sqrt{\Delta P}-R_{\max } f_{w} / 2
$$

where constant coefficient $K$ is equal to 6.72 and $f_{w}$ is the Coriolis force parameters.

Then, the wind speed $v_{s 0}$ at power tower $s$ which locates at the simulated circle of the wind field is defined as

$$
v_{s 0}=\left\{\begin{array}{l}
v_{R \max } r_{s} / R_{\max }, r_{s} \leq R_{\max } \\
v_{R \max }\left(R_{\max } / r_{s}\right)^{\alpha_{v}}, r_{s}>R_{\text {max }}
\end{array}\right.
$$

where $\alpha_{v}$ denotes a constant coefficient within 0.5-0.7 for different typhoon conditions.

If the attenuation of typhoon intensity after landing is considered, the pressure difference after typhoon lands for $t$ hours is represented as

$$
\Delta P(t)=\Delta P_{0}-0.675(1+\sin \varphi) t
$$


where $\Delta P_{0}$ is the pressure difference of the typhoon after landing and $\varphi$ is the angle between the coastline and the direction of typhoon motion.

The impact of the micro-topography on wind speed is ignored in the wind speed information provided by the meteorological department. However, the impact of the micro-topography on wind speed prediction should not be ignored, especially for wind speed in mountain and hillside areas. Based on the result of wind speed prediction, the wind speed around power towers should be corrected according to their locations. The schematic map of power towers' locations is shown in Figure 1, and the correction factors [18] for wind speed around power tower are shown in Table 1.

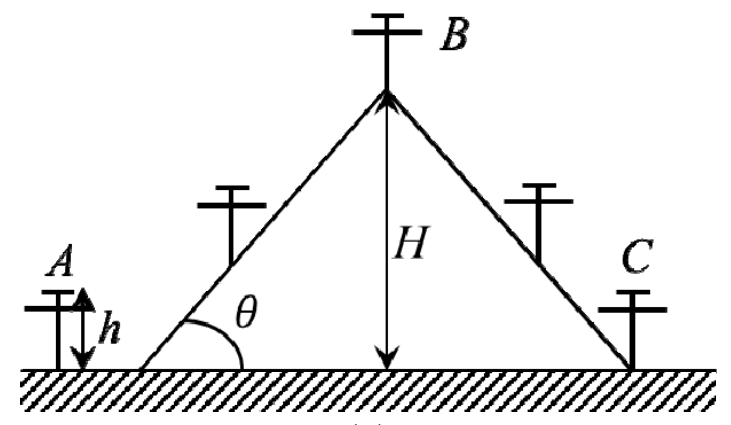

(a)

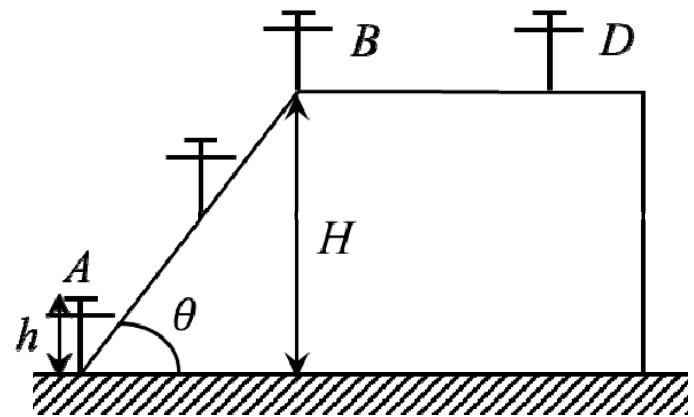

(b)

Figure 1. The schematic map of power towers' locations. (a) mountain; (b) hillside.

Table 1. Correction factors considering the impact of micro-topography.

\begin{tabular}{lc}
\multicolumn{1}{c}{ The Location of Power Tower } & Correction Factor \\
\hline The point $A$ of the windward side in Figure $1 \mathrm{a}$ or in Figure $1 \mathrm{~b}$ & 1 \\
\hline The highest point $B$ in Figure $1 \mathrm{a}$ or in Figure $1 \mathrm{~b}$ & {$\left[1+\kappa \cdot \tan \theta \cdot\left(1-\frac{h}{2.5 H}\right)\right]^{2}$} \\
\hline The windward $A B$ of half way up in Figure $1 \mathrm{a}$ or in Figure $1 \mathrm{~b}$ & $0.5+\left[1+\kappa \cdot \tan \theta \cdot\left(1-\frac{h}{2.5 H}\right)\right]^{2} / 2$ \\
\hline The lee side $B C$ of half way up the hillside in Figure $1 \mathrm{a}$ & $0.25+\left[1+\kappa \cdot \tan \theta \cdot\left(1-\frac{h}{2.5 H}\right)\right]^{2} / 2$ \\
\hline The lee side $C$ in Figure $1 \mathrm{a}$ & 0.5 \\
\hline The dome $D$ in Figure $1 \mathrm{~b}$ & 1 \\
\hline The blocked terrain, such as a mountain basin and a valley & {$[0.75,0.85]$} \\
\hline The valley opening with the same direction as the wind direction & {$[1.20,1.50]$} \\
\hline
\end{tabular}

In Table $1, \tan \theta$ denotes the slope of the windward side of a mountain or a hillside, and is set to 0.3 when it is larger than 0.3 ; constant coefficient $\kappa$ is set to 2.2 and 1.4 for mountain and hillside cases, respectively. $H$ denotes the total height of the mountain or hillside; $h$ denotes the height of the power tower, and is equal to $2.5 \mathrm{H}$ when it is larger than $2.5 \mathrm{H}$ [18].

Considering the impact of the micro-topography on wind speed prediction [18], the wind speed at power tower $s$ is modified, and can be represented as

$$
v_{s}=\sqrt{\beta_{s}} v_{\mathrm{s} 0}
$$

After analyzing the actual damage caused by typhoon in most Southeastern provinces in China, it is shown that the failure rates of the towers could increase obviously with wind speed. Thus, 
the exponential curve function is utilized to describe the relationship between the failure rate $\lambda_{s}$ of power tower $s$ and wind speed $v_{\mathrm{s}}$, and the failure rate [19] is defined as

$$
\lambda_{s}= \begin{cases}0, & 0 \leq v_{s} \leq v_{\text {min }} \\ e^{K_{\lambda}\left(v_{s}-2 v_{\text {min }}\right),}, & v_{\text {min }}<v_{s} \leq 2 v_{\text {min }} \\ 1, & 2 v_{\text {min }}<v_{s}\end{cases}
$$

where $v_{\min }$ is the rated wind speed to prevent the power tower from typhoon. $K_{\lambda}$ denotes a constant coefficient ranging from 0 to 0.4 . Based on the component reliability theory, the failure probability $p_{s}$ of power tower $s$ is defined as

$$
p_{s}=1-e^{-\frac{\lambda_{s}}{1-\lambda_{s}}}
$$

In actual power systems, there are many power towers for supporting a transmission line. The transmission line could be broken if its supporting power towers collapse. Based on the reliability assessment theory of a series system, the predicted failure rate of line $k$ is represented as

$$
E_{k 1}=1-\prod_{s=1}^{N_{\mathrm{k}}}\left(1-p_{s}\right)
$$

where $N_{k}$ is the number of power towers for supporting line $k$.

Considering the hidden failures of lines and the prediction error of typhoon paths, the fault probability of line $k$ is represented as

$$
I_{f p l}\left(L_{k}\right)=\eta E_{k 1}+(1-\eta) E_{k 2}
$$

where $E_{k 2}$ is the average historical fault rate of line $k$ and $\eta$ is a weighting coefficient that is determined by the accuracy of $E_{k 1}$. The greater the value of $I_{f p l}\left(L_{k}\right)$ is, the more important the line $k$ is.

\subsection{Rate of Change of Network Efficiency}

Network efficiency is defined as the mean of the reciprocal of the shortest path length between all node pairs and is utilized to identify the core components of the network topology [20]. Considering the operation state of power systems, the shortest electrical path length $d_{\min , i j}^{X_{L}}$ is adopted [21]. Thus, the network efficiency of a given power system could be represented as

$$
\psi(G)=\frac{1}{m(m-1)} \sum_{1 \leq i<j \leq m} \frac{1}{d_{m i n, i j}^{X_{L}}}
$$

In a power system, removing one line from the power network could lead to the increase of the minimum of lines' reactance and the decrease of the network efficiency. Thus, the importance degree of line $k$ in topology structure of the power system could be determined by the rate of change of network efficiency and can be represented as

$$
I_{r c n}\left(L_{k}\right)=\frac{\psi(G)-\psi\left(G_{-L_{k}}\right)}{\psi(G)}
$$

where $\psi\left(G_{-L_{k}}\right)$ is the network efficiency after line $k$ is removed. It can be concluded that, the smaller the value of $\psi\left(G_{-L_{k}}\right)$ is, the greater the impact of removing line $k$ on the network efficiency is; and, the larger the value of $I_{r c n}\left(L_{k}\right)$ is, the greater the importance degree of line $k$ is. 


\subsection{Cascading Failure Effects of Line Removal on Network Topology}

In an actual power system, line fault could result in the redistribution of power flow. The redistribution of power flow may lead to the overloading of other lines, which could result in cascading failures of lines and then lead to a blackout of the power system. Because the distribution of power flow in a power system is mainly affected by the network topology, the impact of line removal on network topology is analyzed from the perspective of the topology structure, the equivalent structural load $L_{o a}\left(L_{k}\right)$ for line $k$ is defined as

$$
L_{o a}\left(L_{k}\right)=\sum_{i, j \in E} \lambda_{i j}\left(L_{k}\right)
$$

where $\lambda_{i j}\left(L_{k}\right)$ is the number of the shortest electrical paths between node $i$ and node $j$ that pass through line $k$. The greater the number of shortest electrical paths between all node pairs that pass through line $k$ is, the greater the equivalent structural load of line $k$ is.

Considering the limitation of lines' transmission capacity in a real power system, the load bearing function [22] is defined to describe the relationship between the equivalent structural load and the load capacity of the line. The load bearing function of line $k$ at time $t$ is defined as

$$
F_{o a}^{t}\left(L_{k}\right)= \begin{cases}1, & L_{o a}^{t}\left(L_{k}\right) \leq L_{o a}^{0}\left(L_{k}\right) \\ 1+\left(K_{o a}-1\right) \frac{L_{o a}^{t}\left(L_{k}\right)-L_{o o}^{0}\left(L_{k}\right)}{C_{o a}\left(L_{k}\right)-L_{o a}^{0}\left(L_{k}\right)}, & L_{o a}^{0}\left(L_{k}\right)<L_{o a}^{t}\left(L_{k}\right) \leq C_{o a}\left(L_{k}\right) \\ K_{o a}, & C_{o a}\left(L_{k}\right)<L_{o a}^{t}\left(L_{k}\right)\end{cases}
$$

where $L_{o a}^{0}\left(L_{k}\right)$ and $L_{o a}^{t}\left(L_{k}\right)$ are the equivalent structural loads of line $k$ at the initial time and time $t$, respectively; $C_{o a}\left(L_{k}\right)$ is the load capacity of line $k ; K_{o a}$ is the fault influence factor. Three states of the line, i.e., normal state, overload state and failure state, are considered in Equation (14).

It is assumed that $F_{o a}^{0}\left(L_{k}\right)=1(k=1,2, \ldots, n)$ at the initial time. The impact of removing line $k$ is determined by the following steps. Let $F_{o v}^{1}\left(L_{k}\right)=K_{o a}$ and remove line $k$ in the next moment (i.e., $t=1$ ), and the equivalent structural loads and load bearing functions of other lines in the power network after removing line $k$ are determined using Equations (13) and (14). If new lines overload at this time, $t=t+1$, remove the overloaded line with the highest load bearing function from the power system and calculate the load bearing functions of other lines again. This process is repeated until no new overloaded line is caused. If all connected lines of a node fail, the node is removed from the power network. Finally, the cascading failure effects of removal of line $k$ on network topology are defined as

$$
I_{f i l}\left(L_{k}\right)=A_{\text {oal }}\left(L_{k}\right)+A_{\text {oap }}\left(L_{k}\right)
$$

where $A_{\text {oal }}\left(L_{k}\right)$ and $A_{\text {oap }}\left(L_{k}\right)$ are the numbers of the removed lines and nodes caused by overload after the cascading failures triggered by removing line $k$. The greater the value of $I_{f i l}\left(L_{k}\right)$ is, the greater the value of the cascading failure effects on network topology by removing line $k$ is and the more important the line $k$ is.

\subsection{Connected Nodes Importance Degree of Line}

The importance degree of a node is determined by its location in the network topology and the power transmitted by lines connected with the node. Thus, the importance degree of node $i$ is defined as

$$
K_{i}=\delta \frac{\sum_{i_{1}, i_{2} \in E} \lambda_{i_{1} i_{2}}(i)}{\max _{i=1}^{m}\left(\sum_{i_{1}, i_{2} \in E} \lambda_{i_{1} i_{2}}(i)\right)}+(1-\delta) e^{\frac{s_{i}}{m a x} i=1} \frac{\sum_{j \in E_{i(c o n)}}\left|P_{i j}\right|}{\max _{i=1}^{m} \sum_{j \in E_{i(c o n)}}\left|P_{i j}\right|}
$$

where $\lambda_{i_{1} i_{2}}(i)$ denotes the number of shortest electrical paths between node $i_{1}$ and node $i_{2}$ that pass through node $i$ in the power network; $E_{i(c o n)}$ denotes the node set that connects to node $i$ directly; $S_{i}$ is the active power output of node $i$; and $\delta$ is the weight coefficient. Not only the network topology 
(i.e., shortest electrical paths) but also the operation state of the power system is considered by the importance degree of nodes. The larger the number of shortest electrical paths that pass through the node is, the more important in topology the node is; and the greater the connected generators' active power and connected lines' transmission power are, the more important in the operation state the node is.

To include as many critical nodes as possible in the skeleton-network, the lines connected with the critical nodes should be reserved in the skeleton-network. Thus, the connected nodes importance degree of line $k$ is defined as

$$
I_{c i d}\left(L_{k}\right)=\alpha_{c i d} \frac{K_{i}+K_{j}}{2}+\left(1-\alpha_{c i d}\right) \max \left(K_{i}, K_{j}\right)
$$

where $K_{i}$ and $K_{j}$ denote the importance degrees of node $i$ and node $j$ connected by line $k$, respectively; and $\alpha_{c i d}$ denotes a constant coefficient. The more important the nodes connected by line $k$ are, the greater the nodes connection importance degrees of line $k$ is, and the more important the line $k$ is.

\subsection{Weighted Power Flow Betweenness}

The lines in skeleton-network should play important roles in power transmission in a power network, which is beneficial to the subsequent recovery of power supply after disaster caused by extreme weather. Power flow betweenness is adopted to measure the lines' contribution to the active power transmission from generator nodes to load nodes. It overcomes the shortage of the traditional line betweenness index which assumes the power is transmitted along the shortest path [11]. The power flow betweenness is defined as

$$
F_{i j 0}=\sum_{g \in E_{G}} \sum_{d \in E_{D}} \min \left(S_{g}, S_{d}\right) \frac{P_{i j}(g, d)}{P(g, d)}
$$

where $F_{i j 0}$ is the power flow betweenness of line $k$ that connects node $i$ with node $j ; \min \left(S_{g}, S_{d}\right)$ is the minimal value of the active power output $S_{g}$ of generator node $g$ and the active load $S_{d}$ of load node $d$. The values of $P(g, d)$ and $P_{i j}(g, d)$ could be determined by power flow tracing algorithm [23]. $P(g, d)$ and $P_{i j}(g, d)$ are presented as

$$
\begin{gathered}
P(g, d)=\frac{S_{d} S_{g} B_{u d g}^{-1}}{P_{d}} \\
P_{i j}(g, d)=\frac{\left|P_{i j}\right| S_{g} S_{d} B_{u i g}^{-1} B_{d i d}^{-1}}{P_{i} P_{i}}
\end{gathered}
$$

where $P_{d}$ is the sum of active power inflow of node $d ; B_{u d g}^{-1}$ is the element of row $d$ and column $g$ of the inverse of $\boldsymbol{B}_{u} ; B_{u i g}^{-1}$ is the element of row $i$ and column $g$ of the inverse of $\boldsymbol{B}_{u} ; B_{d i d}^{-1}$ is the element of row $i$ and column $d$ of the inverse of $\boldsymbol{B}_{d} ;$ and $P_{i}$ is the sum of the transmitted active power through the lines that connects with node $i$.

Matrix $\boldsymbol{B}_{d}$ and $\boldsymbol{B}_{u}$ are, respectively, determined by downstream and upstream tracing algorithm, and can be represented as

$$
\begin{aligned}
& B_{d i j}=\left\{\begin{array}{l}
1, i=j \\
-\left|P_{i j}\right| / P_{j}, j \in E_{i}^{\text {down }} \\
0, \text { others }
\end{array}\right. \\
& B_{u i j}=\left\{\begin{array}{l}
1, i=j \\
-\left|P_{i j}\right| / P_{j}, j \in E_{i}^{\mathrm{up}} \\
0, \text { others }
\end{array}\right.
\end{aligned}
$$


where $B_{d i j}$ and $B_{u i j}$ are the elements of row $i$ and column $j$ of $\boldsymbol{B}_{d}$ and $\boldsymbol{B}_{u} ; P_{j}$ is the sum of the transmitted active power through the lines that connects to node $j ; E_{i}^{\text {down }}$ is the set of nodes that supplies power to node $i$ directly (i.e., power is transmitted from those nodes to node $i$ through the relevant lines); and $E_{i}^{\mathrm{up}}$ is the set of nodes that is supplied power from node $i$ directly (i.e., power is transmitted from node $i$ to those nodes).

In addition, the larger the transmission capacity of a line is, the larger the margin for the restoration of power supply is. Therefore, the weighted power flow betweenness considering the lines' transmission capacity is employed to measure the importance degree of the line. The weighted power flow betweenness of line $k$ is defined as

$$
I_{w f b}\left(L_{k}\right)=\frac{S_{i j, k}}{\max _{k=1}^{n}\left(S_{i j, k}\right)} F_{i j 0}=\frac{S_{i j, k}}{\max _{k=1}^{n}\left(S_{i j, k}\right)} \sum_{g \in E_{G}} \sum_{d \in E_{D}} \min \left(S_{g}, S_{d}\right) \frac{\left|P_{i j}\right| P_{d} B_{u i g}^{-1} B_{d i d}^{-1}}{P_{i} P_{i} B_{u d g}^{-1}}
$$

The greater the value of $I_{w f b}\left(L_{k}\right)$ is, the more important the line $k$ is from the perspective of the operation state of power systems.

\subsection{Change of Voltage and Reactive Power after Line Removal}

Only the global active power transmission is considered by the weighted power flow betweenness. However, the impact of line removal on local voltage and reactive power should also be considered. In practice, the change of voltage and reactive power after line removal index is proposed to analyze the impact of line removal on voltage and reactive power in power systems [24], and is defined as

$$
\begin{aligned}
& I_{r l c}\left(L_{k}\right)=\max \left(C_{U k}, C_{Q k}\right) \\
& C_{U k}=\left\{\begin{array}{ccc}
1, & \exists U_{i k}>\overline{U_{i c}} \text { or } U_{i k}<\underline{U_{i c},} & i \in E_{D} \\
0, \quad \underline{U_{i c} \leq U_{i k} \leq \overline{U_{i c}}} & i \in E_{D}
\end{array}\right. \\
& C_{Q k}=\left\{\begin{array}{lc}
1, & \exists Q_{i k}-Q_{i 0}>\varepsilon Q_{i c}, \quad i \in E_{G} \\
0, & \text { others }
\end{array}\right.
\end{aligned}
$$

where $C_{U k}$ is utilized to judge whether the nodes' voltages are out of limits after removing line $k$ from the power system; $U_{i k}$ is the voltage of node $i$ after removing line $k ; C_{Q k}$ is utilized to analyze the impact of removing line $k$ on the reactive power of the generator nodes; and $Q_{i 0}$ and $Q_{i k}$ are the reactive power output of generator node $i$ before and after removing line $k$, respectively. If the increased reactive power outputs are higher than $\varepsilon$ times the generator's reactive capacity after removing line $k$, the reactive power regulation of generators are declined obviously, indicating line $k$ is important.

In summary, the influence factors of critical lines identification are comprehensively analyzed by these six indexes from different perspectives. The vulnerable lines under extreme weather such as typhoons are identified by failure probability of line under extreme weather with the parameters of electrical elements and the disaster conditions considered. The network topology structure is considered by rate of change of network efficiency and cascading failure effects of line removal on network topology. The critical nodes are considered by connected nodes importance degree of line. The status of the critical line in the global active power transmission is considered by weighted power flow betweenness, and the impact of line removal on the local voltage and reactive power is considered by change of voltage and reactive power after line removal. The dynamic operation states are considered by these two indexes from the global and local perspectives, respectively. 


\section{Critical Lines Identification Based on the Modified VIKOR Method}

A $p \times n$ line importance degree evaluation matrix is represented as $\boldsymbol{R}=\left[r_{i k}\right]_{p} \times n$ in the critical lines identification for a given power system, where $r_{i k}$ denotes the original value of index $i$ for line $k$, and $p$ denotes the number of the selected evaluation indexes. In this paper, $p$ is equal to 6 . Because the six indexes are with different measurement units, they cannot be integrated for evaluating the importance degrees of the lines directly. These indexes should be normalized before integrating them. For all six indexes, the larger the index value is, the more important the index is. Thus, these indexes can be normalized as

$$
u_{i k}=\frac{r_{i k}-\min _{k=1}^{n}\left(r_{i k}\right)}{\max _{k=1}^{n}\left(r_{i k}\right)-\min _{k=1}^{n}\left(r_{i k}\right)}
$$

where $u_{i k}$ is the normalized value of index $i$ for line $k$.

To make full use of the data characteristic of the values of evaluation indexes and reduce the subjective impacts caused by the experts with different levels of knowledge and experience, a combination weighting method is utilized to determine the weights of indexes with subjective and objective factors considered. Generally, analytic hierarchy process (AHP) [25] and G1 method [26] are adopted for determining the subjective weighting method; criteria importance through interference correlation (CRITIC) [20] and entropy weighting method [27] are adopted for determining the objective weighting. For different evaluation indexes, the importance of subjective and objective weights is different. It is assumed that there are $u$ kinds of subjective weighting methods and $w-u$ kinds of objective weighting methods. $\gamma_{x i}$ is the weight of index $i$ determined by weighting method $x$. Based on the moment estimation theory, the expectation of the subjective and objective weights of index $i$ can be respectively represented as $S_{1}\left(\gamma_{i}\right)$ and $S_{2}\left(\gamma_{i}\right)$, that is

$$
\begin{gathered}
S_{1}\left(\gamma_{i}\right)=\frac{\sum_{x=1}^{u} \gamma_{x i}}{u} \quad i=1,2, \ldots, p \\
S_{2}\left(\gamma_{i}\right)=\frac{\sum_{x=u+1}^{w} \gamma_{x i}}{w-u} \quad i=1,2, \ldots, p
\end{gathered}
$$

Thus, the subjective and objective weighting coefficients for index $i$ can be, respectively, represented as $\tau_{i}$ and $s_{i}$, that is

$$
\begin{aligned}
\tau_{i} & =\frac{S_{1}\left(\gamma_{i}\right)}{S_{1}\left(\gamma_{i}\right)+S_{2}\left(\gamma_{i}\right)} \\
S_{i} & =\frac{S_{2}\left(\gamma_{i}\right)}{S_{1}\left(\gamma_{i}\right)+S_{2}\left(\gamma_{i}\right)}
\end{aligned}
$$

Using the obtained weighting coefficients, and the subjective and objective weights, the synthetic weight $\omega_{i}$ based on the combination weighting method can be represented as

$$
\omega_{i}=\frac{\sum_{x=1}^{u} \tau_{i} \gamma_{x i}+\sum_{x=u+1}^{w} \varsigma_{i} \gamma_{x i}}{\sum_{i=1}^{p}\left(\sum_{x=1}^{u} \tau_{i} \gamma_{x i}+\sum_{x=u+1}^{w} \varsigma_{i} \gamma_{x i}\right)}
$$


The synthetic weights of evaluation indexes are determined by the above combination weighting method, and the next step is to identify critical lines for the skeleton-network. In traditional multi-criteria decision making methods, such as weighted method of simple additivity (SWA) [28] and technique for order preference by similarity to an ideal solution (TOPSIS) [29], only the overall performance of the evaluated object is considered, but the evaluated object' outstanding performance on single index is seldom considered. In practice, the VIKOR method is a compromise method to solve decision problems with conflicting complex systems, which has been widely applied in multi-attribute decision-making problems [30,31]. Minimizing individual regret and maximizing group utility are taken as the decision objectives in the VIKOR method. For a multi-indexes decision-making problem with an evaluation matrix $\boldsymbol{R}=\left[r_{i k}\right]_{p \times n}$, the comprehensive evaluation result is determined by the VIKOR method, that is

$$
Q_{k}=\mu \frac{S_{k}-\min _{k}^{n} S_{k}}{\max _{k}^{n} S_{k}-\min _{k}^{n} S_{k}}+(1-\mu) \frac{R_{k}-\min _{k}^{n} R_{k}}{\max _{k}^{n} R_{k}-\min _{k}^{n} R_{k}}
$$

where $Q_{k}$ denotes the comprehensive evaluation result of object $k$, i.e., line $k$ for critical lines identification. The smaller the $Q_{k}$ is, the higher the line $k$ ranks. $\mu$ is the decision-making coefficient. $S_{k}$ denotes the group utility of line $k$ and is represented as

$$
S_{k}=\sum_{i=1}^{p}\left[\omega_{i}\left(\max _{k}^{n} r_{i k}-r_{i k}\right) /\left(\max _{k}^{n} r_{i k}-\min _{k}^{n} r_{i k}\right)\right]
$$

$R_{k}$ denotes the individual regret of line $k$ and is represented as

$$
R_{k}=\max _{i=1}^{p}\left[\left(\max _{k}^{n} r_{i k}-r_{i k}\right) /\left(\max _{k}^{n} r_{i k}-\min _{k}^{n} r_{i k}\right)\right]
$$

The worst index values of the objects (i.e., lines) in the individual regret are considered in Equation (35) by using the VIKOR method. In the skeleton-network optimization, the objective is to include critical lines with important function in a particular aspect, which is opposite to the individual regret in Equation (35). Thus, Equation (35) is modified for critical lines identification, that is

$$
R_{k}^{\prime}=\min _{i=1}^{p}\left[\left(\max _{k}^{n} r_{i k}-r_{i k}\right) /\left(\max _{k}^{n} r_{i k}-\min _{k}^{n} r_{i k}\right)\right]
$$

In the modified VIKOR method, maximizing outstanding individual performance and group utility is taken as the objective of critical lines identification. Not only the comprehensive performance of the concerned indexes of the lines is considered, but also the impacts of line removal on certain aspects of power systems are highlighted. The smaller the value of $S_{k}$ is, the greater the group benefit is, i.e., the better the overall performance of line $k$ is; the smaller the value of $R_{k}^{\prime}$ is, the greater the outstanding individual performance is, i.e., the better the outstanding performance of line $k$ on a particular aspect is; and the smaller the value of $Q_{k}$ is, the better both the group benefit and the individual performance are, i.e., the higher the line $k$ ranks.

Thus, the flowchart of critical lines identification for the skeleton-network is shown in Figure 2. 


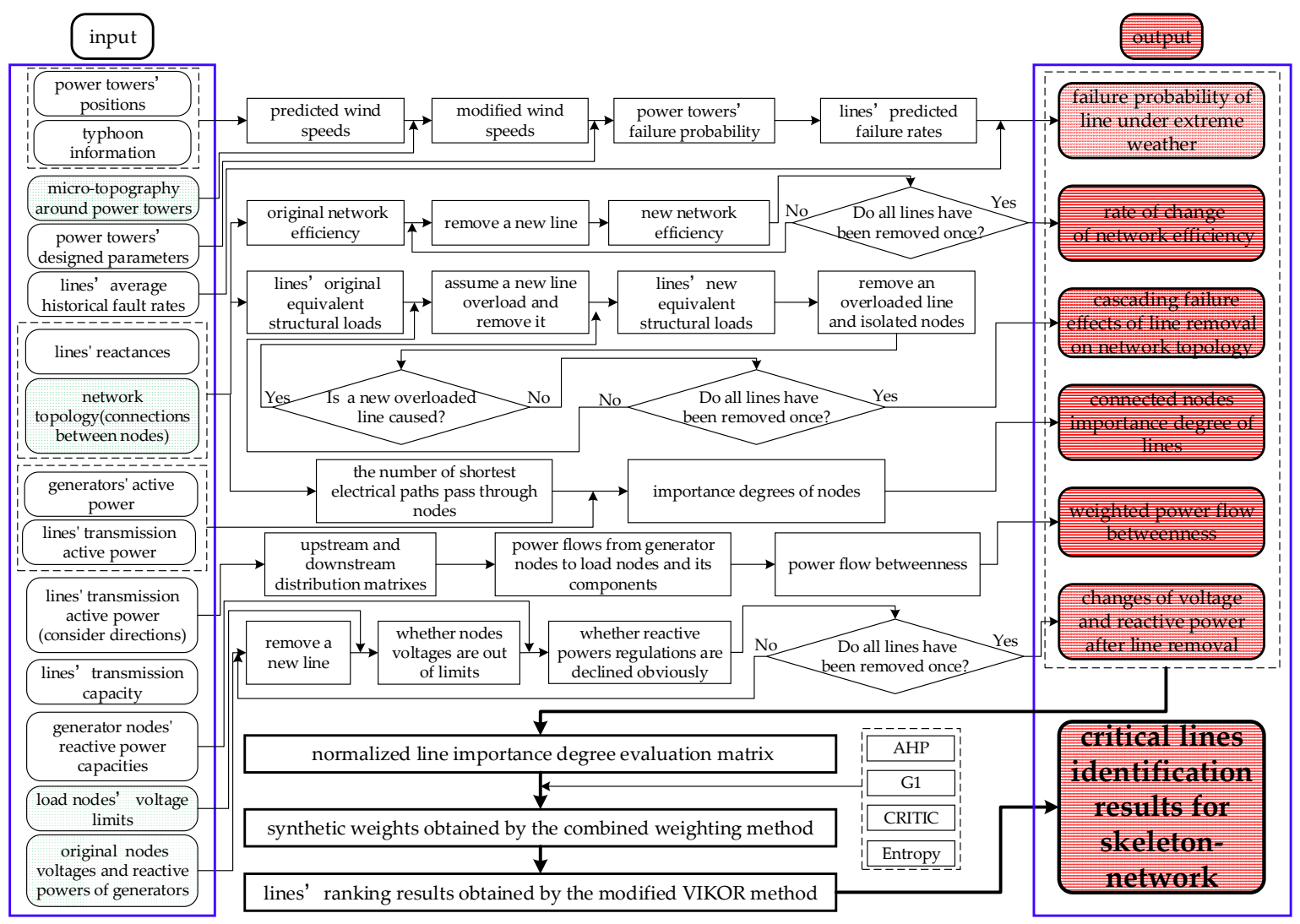

Figure 2. The flowchart of critical lines identification for the skeleton-network.

\section{Numerical Results}

\subsection{Case Study}

Part of the Guangdong power system in China (Figure 3), including 155 transmission lines and 125 nodes, is utilized to verify the effectiveness of the proposed multi-attribute evaluation method based on the modified VIKOR method (MMVIK) for critical lines identification. In an actual regional power system of China, its subsystems with different voltage levels are regulated by different levels of power grid corporations. The lines with $500 \mathrm{kV}$ voltage level in the power system are managed by the provincial power grid corporation, so these lines are ignored by the regional power grid corporations which are the subsidiaries of the provincial one. The results of critical lines identification are determined by the lines' importance degree evaluation results and the actual demands of power grid corporations. In this paper, the top ten lines obtained by using the MMVIK method are identified as critical lines for easy demonstration. It is assumed that $\Delta p=935 \mathrm{hPa}, v_{T}=5.6 \mathrm{~m} / \mathrm{s}, v_{\min }=35 \mathrm{~m} / \mathrm{s}$, $\eta=0.8, K_{\lambda}=0.1, \alpha_{\text {cid }}=0.5, \delta=0.8, \chi=0.9$ and $\mu=0.6$ [19].

The correlation between the evaluation indexes is needed to be analyzed first to verify whether the index selection is reasonable. Spearman correlation coefficient is adopted to analyze the correlation between the six indexes because it is not very strict on data qualification and can analyze the correlation by the ranking position of indexes' values, which is suitable for the critical lines identification. Spearman correlation coefficients between any two indexes are shown in Table 2. It can be seen in Table 2 that most of the correlations between the evaluation indexes are weak except for the pair of indexes $I_{r c n}$ and $I_{c i d}$, because lines' position in network topology is considered in $I_{r c n}$ and nodes' position in network topology is considered in $I_{c i d}$. However, the objective of $I_{r c n}$ is to identify critical lines which locate in the center of the network, and the objective of $I_{\text {cid }}$ is to include critical nodes which are important for both network topology and operation state of power systems. Thus, these two 
indexes are different with each other actually. It can be concluded that there is not much overlap and cross information among the selected indexes and the selection of these indexes is reasonable.

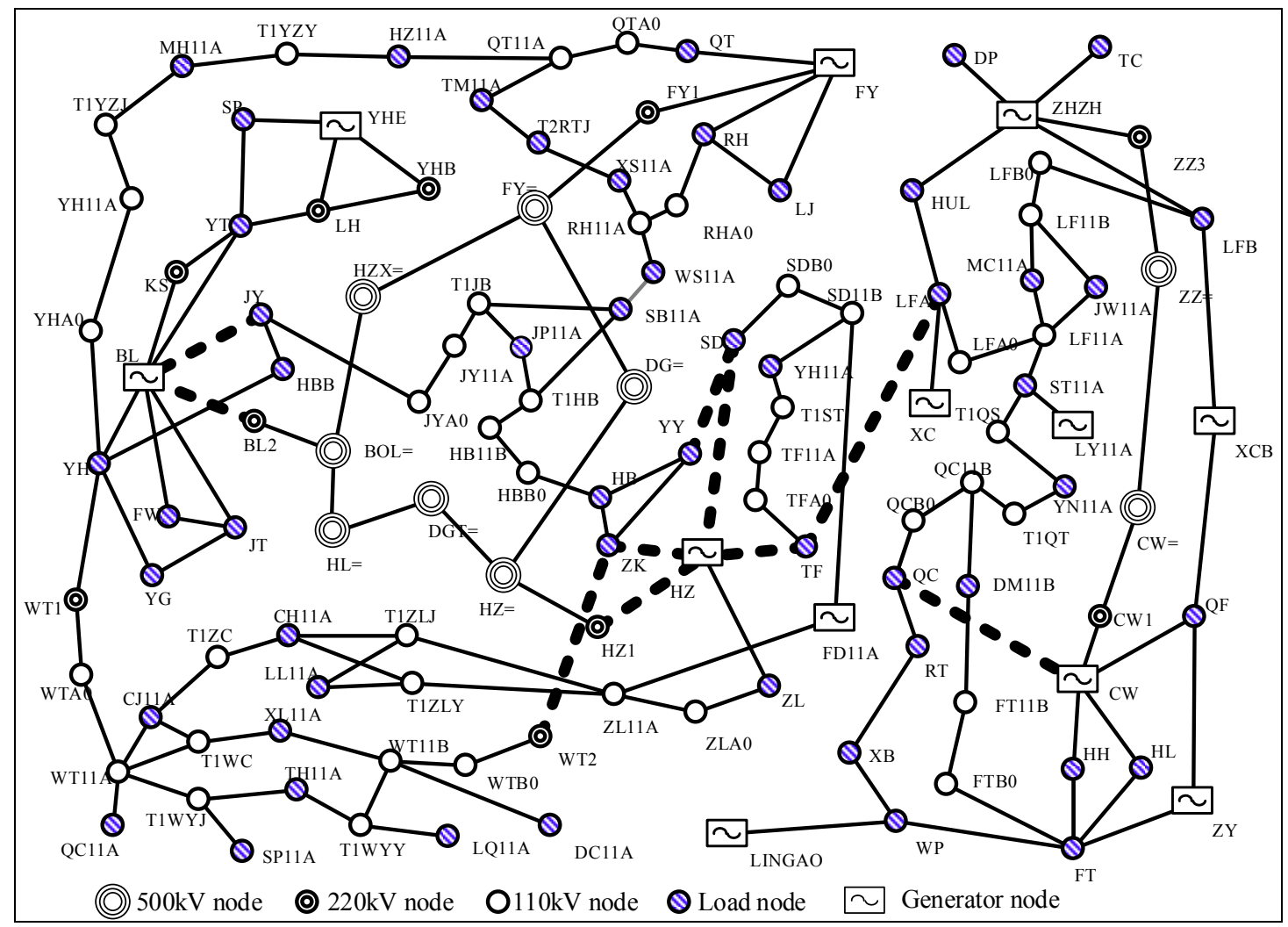

Figure 3. Diagram of a regional power system in Guangdong.

Table 2. Spearman correlation coefficients between any two evaluation indexes.

\begin{tabular}{ccccccc}
\hline Correlation Coefficient & $\boldsymbol{I}_{\boldsymbol{f} \boldsymbol{p l}}$ & $\boldsymbol{I}_{\boldsymbol{r c n}}$ & $\boldsymbol{I}_{\boldsymbol{f i l}}$ & $\boldsymbol{I}_{\boldsymbol{c i d}}$ & $\boldsymbol{I}_{\boldsymbol{w f b} \boldsymbol{b}}$ & $\boldsymbol{I}_{\boldsymbol{r l c}}$ \\
\hline$I_{f p l}$ & 1.000 & -0.068 & 0.321 & 0.079 & -0.077 & 0.118 \\
$I_{r c n}$ & -0.068 & 1.000 & 0.022 & 0.595 & 0.199 & 0.089 \\
$I_{f i l}$ & 0.321 & 0.022 & 1.000 & 0.101 & 0.114 & 0.039 \\
$I_{c i d}$ & 0.079 & 0.595 & 0.101 & 1.000 & 0.087 & 0.134 \\
$I_{w f f b}$ & -0.077 & 0.199 & 0.114 & 0.087 & 1.000 & 0.067 \\
$I_{r l c}$ & 0.118 & 0.089 & 0.039 & 0.134 & 0.067 & 1.000 \\
\hline
\end{tabular}

After identification, the top ten lines are determined as the lines with bold dotted lines in Figure 3, as also shown in Table 3. It can be seen in Table 3 that the selected evaluation indexes have similarities and complementarity. For example, the values of $I_{r c n}$ and $I_{r l c}$ for $L_{T F-L F A}$ are 1 , which indicates that some critical lines locate at the center of the power network topology; and the nodes voltage might be out of limits or the reactive power regulation of generators might be declined after removing line $k$. $L_{H Z-Z K}$ is with a smaller $I_{r c n}$ (i.e., 0.034 ) and a relatively bigger $I_{w f f b}$ (i.e., 0.531 ), indicating that $L_{H Z-Z K}$ plays a less important role in topology but is important for power transmission. In addition, if a single index (e.g., $I_{r c n}$ ) is utilized to evaluate line importance degree, some critical lines would be ignored for critical lines identification. For instance, the value of $I_{r c n}$ for $L_{H Z-S D}$ is 0.038 , which ranks in the 35th based on $I_{r c n}$ only, $L_{\mathrm{HZ}-\mathrm{SD}}$ could not be identified as a critical line because it is not in the center of network topology. However, the value of $I_{w f f}$ for $L_{H Z-S D}$ is 0.572 , which ranks 4 th based on $I_{w f f}$ only, indicating $L_{H Z-S D}$ has a great influence on the operation state of the power system; the value of $I_{\text {fil }}$ for $L_{\mathrm{HZ}-\mathrm{SD}}$ is 1 , indicating removing $L_{\mathrm{HZ}-\mathrm{SD}}$ might cause a bigger cascading failure. Thus, $L_{H Z-S D}$ should 
be identified as a critical line. As a result, the key characteristic parameters of the power systems can be considered more reasonably and comprehensively by integrating the six indexes, reducing the probability of missing some critical lines to a certain extent.

Table 3. The normalized result of the top ten lines' evaluation indexes.

\begin{tabular}{cccccccc}
\hline Ranking & Line & $\boldsymbol{I}_{\boldsymbol{f} \boldsymbol{l} \boldsymbol{l}}$ & $\boldsymbol{I}_{\boldsymbol{r c n}}$ & $\boldsymbol{I}_{\boldsymbol{f i l}}$ & $\boldsymbol{I}_{\boldsymbol{c i d}}$ & $\boldsymbol{I}_{\boldsymbol{w f b} \boldsymbol{b}}$ & $\boldsymbol{I}_{\boldsymbol{r l c}}$ \\
\hline 1 & $L_{T F-L F A}$ & 0.030 & 1.000 & 0.000 & 0.656 & 0.330 & 1 \\
2 & $L_{H Z-H Z 1}$ & 0.059 & 0.728 & 0.700 & 1.000 & 1.000 & 0 \\
3 & $L_{B L-B L 2}$ & 0.063 & 0.508 & 0.500 & 0.720 & 0.524 & 1 \\
4 & $L_{C W-Q C}$ & 1.000 & 0.050 & 0.500 & 0.305 & 0.605 & 0 \\
5 & $L_{H Z-T F}$ & 0.868 & 0.617 & 0.300 & 0.931 & 0.005 & 0 \\
6 & $L_{H Z-Z K}$ & 0.293 & 0.034 & 1.000 & 0.794 & 0.531 & 0 \\
7 & $L_{H Z-S D}$ & 0.090 & 0.038 & 1.000 & 0.699 & 0.572 & 0 \\
8 & $L_{Y Y-S D}$ & 0.078 & 0.034 & 0.700 & 0.056 & 0.242 & 1 \\
9 & $L_{B L-J Y}$ & 0.262 & 0.036 & 0.500 & 0.501 & 0.092 & 1 \\
10 & $L_{Z K-W T 2}$ & 0.868 & 0.139 & 0.200 & 0.253 & 0.029 & 0 \\
\hline
\end{tabular}

Table 4 shows the results of indexes weights calculated by single weighting methods and the proposed combination weighting method. It can be seen in Table 4 that the weights of $I_{\text {fil }}$ and $I_{\text {cid }}$ determined by the entropy weighting method are 0.0549 and 0.0616 , respectively, and then $I_{\text {fil }}$ and $I_{c i d}$ are neglected in critical lines identification. The objective weights of these two indexes are relatively small because the difference of the indexes values is small and their weights obtained by the objective weighting method are based on data characteristics. Thus, the objective weighting results might be unreasonable because the objective weighting method ignores the experts' experience. On the other hand, with the subjective impacts caused by the experts with different levels of knowledge and experience considered, it is not scientific to rely on the subjective weights given by the experts results only. In the proposed combination weighting method, the synthetic weights of $I_{r c n}, I_{f i l}$ and $I_{c i d}$ are $0.1163,0.0950$ and 0.1146 , respectively. These three indexes' weights are relatively small, which is reasonable because $I_{r c n}$ and $I_{f i l}$ are utilized to evaluate the importance degrees of lines from the pure topology, and the difference between the values of $I_{\text {fil }}$ and $I_{\text {cid }}$ is small. Therefore, the proposed combination weighting method that can consider both the data characteristic and experts' experience is more suitable for determining the indexes' weights for the critical lines identification.

Table 4. Weights of each evaluation index under different weighting methods.

\begin{tabular}{cccccc}
\hline \multirow{2}{*}{$\begin{array}{c}\text { Evaluation } \\
\text { Method }\end{array}$} & \multicolumn{2}{c}{ Subjective Weighting Method } & \multicolumn{2}{c}{ Objective Weighting Method } & $\begin{array}{c}\text { Combination } \\
\text { Weighting Method }\end{array}$ \\
\cline { 2 - 4 } & AHP & G1 & CRITIC & Entropy & 0.2221 \\
$I_{f p l}$ & 0.3255 & 0.2854 & 0.1535 & 0.1375 & 0.1163 \\
$I_{r c n}$ & 0.0940 & 0.0843 & 0.1173 & 0.1982 & 0.0950 \\
$I_{f i l}$ & 0.0862 & 0.1214 & 0.1710 & 0.0549 & 0.1146 \\
$I_{c i d}$ & 0.1356 & 0.1699 & 0.1318 & 0.0616 & 0.1912 \\
$I_{w f b}$ & 0.2848 & 0.2379 & 0.1266 & 0.1462 & 0.2608 \\
$I_{r l c}$ & 0.0740 & 0.1011 & 0.2998 & 0.4015 & \\
\hline
\end{tabular}

For the top ten lines, three parameters of the MMVIK method, i.e., group utility $S_{k}$, individual performance $R_{k}$ and comprehensive evaluation $Q_{k}$, are shown in Table 5 . It can be seen in Table 5 that the values of $S_{k}$ for both $L_{H Z-Z K}$ and $L_{H Z-S D}$ are bigger than those for $L_{Y Y-S D}$ and $L_{B L-J Y}$, indicating the overall performances of $L_{H Z-Z K}$ and $L_{H Z-S D}$ are not as good as those of $L_{Y Y-S D}$ and $L_{B L-J Y}$. However, the values of $Q_{k}$ for $L_{H Z-Z K}$ and $L_{H Z-S D}$ are smaller than those for $L_{Y Y-S D}$ and $L_{B L-J Y}$ because the values of $R_{k}$ for $L_{H Z-Z K}$ and $L_{H Z-S D}$ are smaller than those for $L_{Y Y-S D}$ and $L_{B L-J Y}$. It can be seen in Table 3 that both $L_{H Z-Z K}$ and $L_{H Z-S D}$ are with big values of $I_{f i l}$ (i.e., 1 ), which indicates that removal of them might cause a bigger cascading failure of the power system. In other words, $L_{H Z-Z K}$ and $L_{H Z-S D}$ play 
important roles in a particular aspect of the power system. It also can be seen from Table 3 that most of the values of the six indexes for both $L_{Y Y-S D}$ and $L_{B L-J Y}$ is not large. Thus, it is more reasonable that $L_{H Z-Z K}$ and $L_{H Z-S D}$ rank slightly higher than $L_{Y Y-S D}$ and $L_{B L-J Y}$ in the MMVIK method. Therefore, the MMVIK method is effective in identifying critical lines for the skeleton-network which wants to contain a variety of critical lines.

Table 5. Three parameters of the multi-attribute evaluation method based on the modified VIKOR method (MMVIK) method for the top ten lines.

\begin{tabular}{ccccc}
\hline Ranking & Line & $\boldsymbol{S}_{\boldsymbol{k}}$ & $\boldsymbol{R}_{\boldsymbol{k}}$ & $\boldsymbol{Q}_{\boldsymbol{k}}$ \\
\hline 1 & $L_{T F-L F A}$ & 0.4781 & 0 & 0.0466 \\
2 & $L_{H Z-H Z 1}$ & 0.5298 & 0 & 0.1038 \\
3 & $L_{B L-B L 2}$ & 0.4359 & 0.2805 & 0.1187 \\
4 & $L_{C W-Q C}$ & 0.5740 & 0 & 0.1526 \\
5 & $L_{H Z-T F}$ & 0.5994 & 0.0692 & 0.2100 \\
6 & $L_{H Z-Z K}$ & 0.6434 & 0 & 0.2293 \\
7 & $L_{H Z-S D}$ & 0.6912 & 0 & 0.2822 \\
8 & $L_{Y Y-S D}$ & 0.5987 & 0.3000 & 0.3069 \\
9 & $L_{B L-J Y}$ & 0.5543 & 0.4219 & 0.3094 \\
10 & $L_{Z K-W T 2}$ & 0.7376 & 0.1323 & 0.3894 \\
\hline
\end{tabular}

\subsection{Comparisons with Other Methods}

To further demonstrate the effectiveness of the MMVIK method for critical lines identification, five methods, i.e., single-attribute evaluation method based on the rate of change of network efficient (SRCNE) [8], single-attribute evaluation method based on hybrid flow betweenness (SHFB) [11], multi-attribute evaluation method based on utility theory and cooperative game (MUTCG) [15], SWA [28] and TOPSIS [29], are employed for comparisons. The final line importance evaluation results and the top ten lines of the actual regional Guangdong power system obtained by the six methods are shown in Figure 4 and Table 6, respectively.

It can be seen in Figure 4 that most of the critical lines identified by single-attribute evaluation methods are included in the identification results obtained by the proposed MMVIK method, such as line 27 (i.e., $L_{H Z-T F}$ ), line 50 (i.e., $L_{T F-L F A}$ ), line 60 (i.e., $L_{B L-B L 2}$ ) and line 61 (i.e., $L_{H Z-H Z 1}$ ) identified by SRCNE method. However, some critical lines are ignored in single-attribute evaluation methods (i.e., SRCNE and SHFB), such as line 24 (i.e., $L_{H Z-Z K}$ ). Line 24 connects central node $H Z$ with node $Z K$, and it is the main channel to transmit power from the generator node $H Z$ to load nodes. Thus, it is more reasonable to identify line 24 as a critical line with higher importance degree by the MMVIK method. In the MUTCG method, only network topology and the power transmitted on the lines are considered, and the lines' failure probability and the impact of line removal are ignored. For example, line 31 (i.e., $L_{Z K-W T 2}$ ) with a big value of $I_{f p l}$ (i.e., 0.868 ), which is easy to fail under typhoon, should be involved in reinforcement work to reduce lost caused by extreme weather. Line 28 (i.e., $L_{Y Y-S D}$ ) with a big value of $I_{\text {fil }}$ (i.e., 0.7 ), which has a great impact on other lines and is the main channel to transmit power from the generator node $\mathrm{HZ}$ to load nodes, should have a relatively high importance degree. The nodes voltages might be out of limits or the reactive power regulations of generators might be declined obviously after removing line 2 (i.e., $L_{B L-J Y}$ ) with a big value of $I_{r l c}$ (i.e., 1 ). However, these three lines rank 27th, 29th and 18th with a relatively low importance degree when using the MUTCG method. Therefore, it can be concluded that the proposed MMVIK method is better than these three methods for critical lines identification in the skeleton-network. 
(a) MMVIK(this paper)

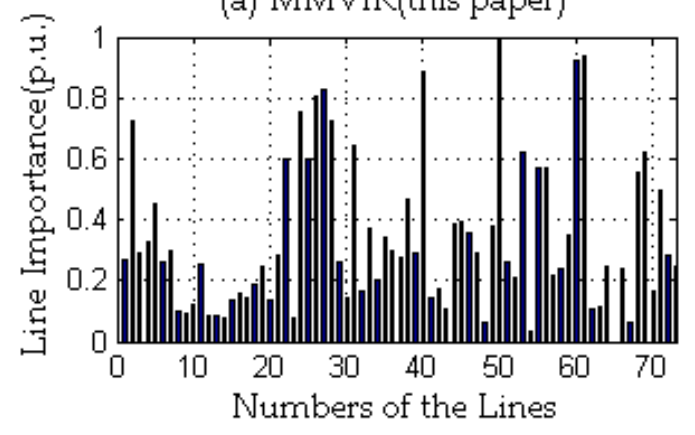

(c) SHFB Method

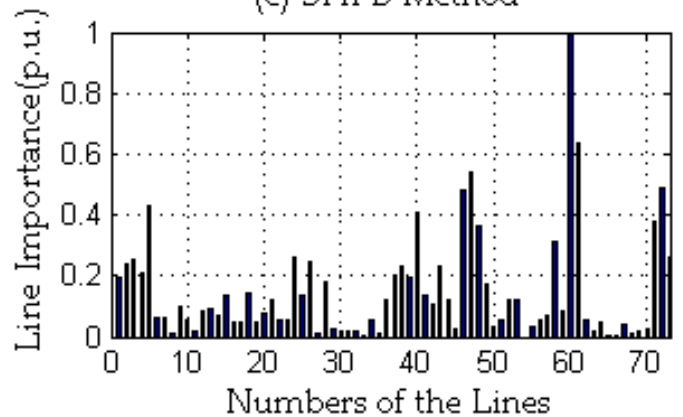

(e) SWA Method

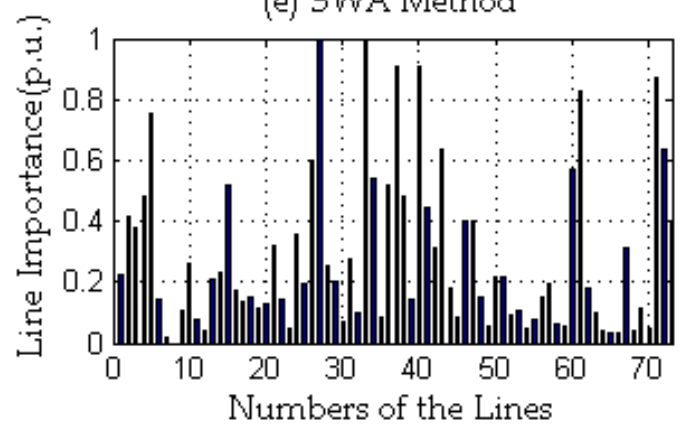

(b) SRCNE Method

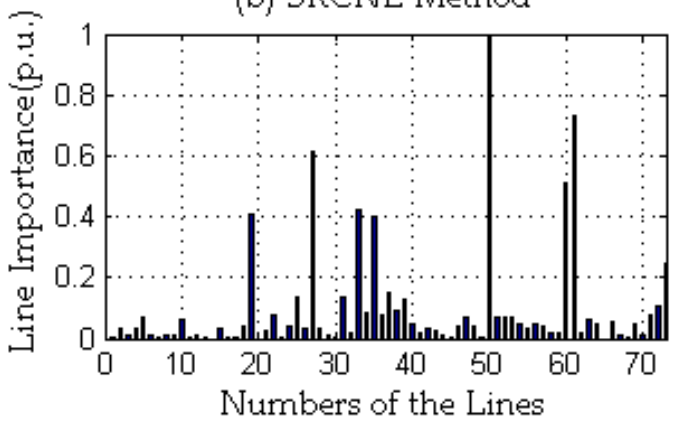

(d) MUTCG Method

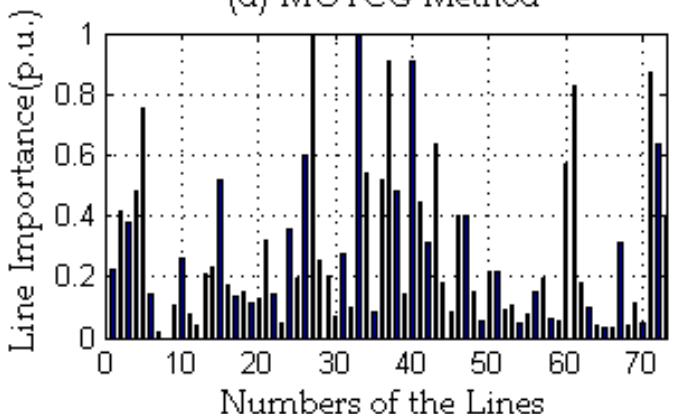

(f) TOPSIS Method

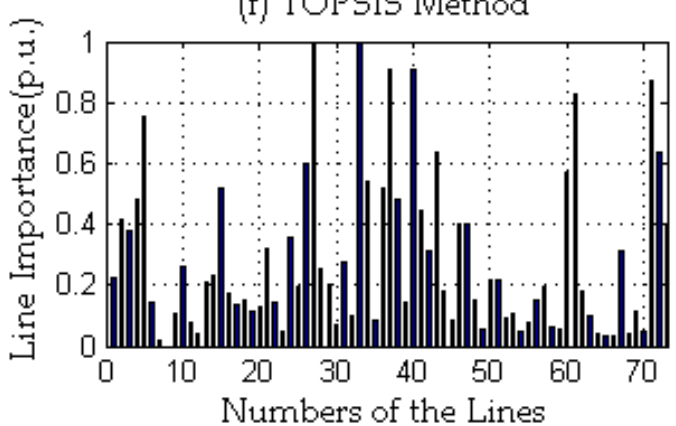

Figure 4. The lines importance degrees identified by the multi-attribute evaluation method based on the modified VIKOR method (MMVIK), single-attribute evaluation method based on the rate of change of network efficient (SRCNE), single-attribute evaluation method based on hybrid flow betweenness (SHFB), multi-attribute evaluation method based on utility theory and cooperative game (MUTCG), weighted method of simple additivity (SWA) and technique for order preference by similarity to an ideal solution (TOPSIS) methods.

Table 6. The top ten lines identified by the MMVIK, SRCNE, SHFB, MUTCG, SWA, and TOPSIS methods.

\begin{tabular}{ccccccc}
\hline Ranking & MMVIK & SRCNE & SHFB & MUTCG & SWA & TOPSIS \\
\hline 1 & $L_{T F-L F A}$ & $L_{T F-L F A}$ & $L_{B L-B L 2}$ & $L_{T F-L F A}$ & $L_{B L-B L 2}$ & $L_{H Z-H Z 1}$ \\
2 & $L_{H Z-H Z 1}$ & $L_{H Z-H Z 1}$ & $L_{H Z-H Z 1}$ & $L_{H U L-L F A}$ & $L_{T F-L F A}$ & $L_{T F-L F A}$ \\
3 & $L_{B L-B L 2}$ & $L_{H Z-T F}$ & $L_{F T-W P}$ & $L_{H Z-H Z 1}$ & $L_{H Z-H Z 1}$ & $L_{H Z-T F}$ \\
4 & $L_{C W-Q C}$ & $L_{B L-B L 2}$ & $L_{C W 1-C W}$ & $L_{B L-J Y}$ & $L_{B L-J Y}$ & $L_{B L-B L 2}$ \\
5 & $L_{H Z-T F}$ & $L_{H U L-L F A}$ & $L_{Z Y-F T}$ & $L_{C W-Q C}$ & $L_{C W-Q C}$ & $L_{C W-Q C}$ \\
6 & $L_{H Z-Z K}$ & $L_{Y H E-Y H B}$ & $L_{B L-Y H}$ & $L_{Y Y-S D}$ & $L_{Y Y-S D}$ & $L_{H Z-Z K}$ \\
7 & $L_{H Z-S D}$ & $L_{Z H Z H-H U L}$ & $L_{C W-Q C}$ & $L_{H Z-T F}$ & $L_{H Z-T F}$ & $L_{H Z-S D}$ \\
8 & $L_{Y Y-S D}$ & $L_{F Y 1-F Y}$ & $L_{Z H Z H-Z Z 3}$ & $L_{Z H Z H-T C}$ & $L_{Z Z-T C}$ & $L_{Z K-W T 2}$ \\
9 & $L_{B L-J Y}$ & $L_{Q F-X C}$ & $L_{X B-W P}$ & $L_{H Z-Z K}$ & $L_{H Z-Z K}$ & $L_{H Z-Z L}$ \\
10 & $L_{Z K-W T 2}$ & $L_{Z K-W T 2}$ & $L_{J Y-J Y A 0}$ & $L_{L F A-L F A 0}$ & $L_{L F A-L F A 0}$ & $L_{B L-J Y}$ \\
\hline
\end{tabular}


It can be seen in Table 6 that the top ten lines identified by the MMVIK, SWA and TOPSIS methods are similar, but their ranking order is slightly different. $L_{T F-L F A}$ ranks first by using the proposed MMVIK while $L_{B L-B L 2}$ and $L_{H Z-H Z 1}$ rank first by using the SWA and TOPSIS methods, respectively. It can be seen in Figure 3 that $L_{\text {TF-LFA, }}$ which locates at the center of the network topology, is the sole interconnected line between two subsystems, and it has relatively high values of $I_{c i d}$ and $I_{r l c}$ (i.e., 1). The power system could be split into two isolated islands and the voltage and the reactive power could also be affected after the removal of $L_{\text {TF-LFA }}$. It can also be seen in Figure 3 that both the location in the network topology and the function for power transmission of $L_{B L-B L 2}$ and $L_{H Z-H Z 1}$ are similar. If any of them failed, the power flow can be transmitted through the other line. Thus, the impact of removing $L_{T F-L F A}$ is bigger than removing $L_{B L-B L 2}$ or $L_{H Z-H Z 1}$ and it is more reasonable to rank $L_{T F-L F A}$ as the first one. In addition, $L_{H Z-S D}$ and $L_{Z K-W T 2}$ are identified as the critical lines by the MMVIK method. It can be seen in Table 3 that removing $L_{H Z-S D}$ from the network could lead to a cascading failure for the power system because the value of $I_{\text {fil }}$ for $L_{H Z-S D}$ is equal to 1 , and $L_{Z K-W T 2}$ is easy to fail under typhoon because the value of $I_{f p l}$ for $L_{H Z-S D}$ is equal to 0.868 , so these two lines should be identified as the critical lines with high ranking orders. However, $L_{H Z-S D}$ and $L_{Z K-W T 2}$ are not identified as critical lines by the SWA method. It can be concluded that the MMVIK method not only takes the comprehensive performance of the lines under various indexes into account, but also considers the outstanding contribution of some important lines on a particular aspect of the power system, which indicates that it is a better method to identify critical lines for the skeleton-network.

\section{Conclusions}

Six indexes, i.e., failure probability of line under extreme weather, rate of change of network efficiency, cascading failure effects of line removal on network topology, connected nodes importance degree of line, weighted power flow betweenness, and change of voltage and reactive power after line removal, are first presented for identifying critical lines for the skeleton-network from the perspective of the lines' parameter and extreme weather, the network topology and the operation state of power systems. Then, the line importance degree evaluation is represented as a multi-indexes decision-making model. The modified VIKOR method is presented to identify the importance degrees of lines based on the synthetic weights obtained by the combination weighting method. Numerical results of case study on a part of the actual Guangdong power system in China show that the proposed method is reasonable and effective. The proposed multi-indexes consider the key characteristic parameters of the complex power system, thus are more comprehensive than the single-attribute evaluation index (i.e., SRCNE and SHFB) for critical lines identification. The proposed combination weighting method considers data characteristic and experts' experience, and is more reasonable than single weighting method (e.g., entropy weighing method) for determining the weights of the indexes. The modified VIKOR method, which considers the overall performance and the outstanding individual performance of lines comprehensively, is better than the MUTCG method and the traditional comprehensive evaluation method (i.e., SWA and TOPSIS). Only the impacts of typhoon on critical lines identification is considered in this paper, thus the impacts of other extreme weather conditions such as ice and rainstorm disasters could be considered in future research.

Author Contributions: C.H. and Z.L. conceptualized the study; Y.Z. and Z.L. performed the analyses; G.L., T.M. and X.Y. acquired funding; C.H. performed investigations; G.L., T.M. and X.Y. acquired resources; C.H. wrote the original draft; and Y.Z., Z.L., Y.D. and L.Y. reviewed and edited the manuscript.

Acknowledgments: This work was supported by Zhejiang Provincial Natural Science Foundation of China (LY17E070003), National Natural Science Foundation of China (51377005 and 51777185) and a project from Guangdong Power Grid Corporation (GDKJQQ20153014).

Conflicts of Interest: The authors declare no conflict of interest. 


\section{Nomenclature}

$I_{f p l}\left(L_{k}\right) \quad$ The fault probability of line $k$ (in p.u.)

$I_{r c n}\left(L_{k}\right) \quad$ The rate of change of network efficiency of line $k$ (in p.u.)

$I_{\text {fil }}\left(L_{k}\right) \quad$ The cascading failure effects of removal of line $k$ on network topology (in p.u.)

$I_{\text {cid }}\left(L_{k}\right) \quad$ The connected nodes importance degree of line $k$ (in p.u.)

$I_{\text {wofb }}\left(L_{k}\right) \quad$ The weighted power flow betweenness of line $k$ (in p.u.)

$I_{r l c}\left(L_{k}\right) \quad$ The change of voltage and reactive power after removal of line $k$ (in p.u.)

$m \quad$ The number of nodes

$n \quad$ The number of lines

$R_{\max } \quad$ The maximum wind radius (in $\mathrm{m}$ )

$\Delta P \quad$ The pressure difference between the outer periphery of tropical cyclone and the typhoon center (in $\mathrm{hPa}$ )

$\beta_{s} \quad$ The correction factor for wind speed around power tower $s$ (in p.u.)

$r_{s} \quad$ The distance between tower $s$ and typhoon center (in $\mathrm{m}$ )

$v_{s} \quad$ The modified wind speed at power tower $s$ (in $\mathrm{m} / \mathrm{s}$ )

$d_{\text {min,ij }}^{X_{L}} \quad$ The minimum sum of lines' reactance in the path between node $i$ and node $j$ (in p.u.)

$\psi(G) \quad$ The network efficiency of a given power system $G$ (in p.u.)

$L_{k} / L_{i-j} \quad$ Line $k$ that connects node $i$ with node $j$

$L_{o a}\left(L_{k}\right) \quad$ The equivalent structural load for line $k$ (in p.u.)

$F_{\text {oa }}^{t}\left(L_{k}\right) \quad$ The load bearing function of line $k$ at time $t$ (in p.u.)

$K_{i} \quad$ The importance degree of node $i$ (in p.u.)

$E_{G}, E_{D} \quad$ The generator and load node sets

$P(g, d) \quad$ The power flow from generator node $g$ to load node $d$ (in MW)

$P_{i j}(g, d) \quad$ The components of $P(g, d)$ on line $L_{i-j}$ (in MW)

$\boldsymbol{B}_{u}, \boldsymbol{B}_{d} \quad$ The upstream and downstream distribution matrixes (in p.u.)

$P_{i j} \quad$ The active power of line $L_{i-j}$ (in MW)

$S_{i j, k} \quad$ The transmission capacity of line $k$ which connects node $i$ with node $j$ (in MW)

$\overline{U_{i c}}, U_{i c} \quad$ The upper and lower voltage limits of generator node $i$ (in $\mathrm{kV}$ )

$Q_{i c} \quad$ The reactive power capacity of generator node $i$ (in MVA)

$\omega_{i} \quad$ The integrated weight of index $i$ (in p.u.)

\section{References}

1. Huang, J.H.; Ge, S.Y.; Han, J.; Li, H.; Zhou, X.M.; Liu, H.; Wang, B.; Chen, Z.F. A diagnostic method for distribution networks based on power supply safety standards. Prot. Control Mod. Power Syst. 2016, 1, 1-8. [CrossRef]

2. Bai, F.F.; Liu, Y.; Liu, Y.L.; Sun, K.; Bhatt, N.; Rosso, A.D.; Farantatos, E. A measurement-based approach for power system instability early warning. Prot. Control Mod. Power Syst. 2016, 1, 17-25. [CrossRef]

3. Yang, Y.; Nishikawa, T.; Motter, A.E. Small vulnerable sets determine large network cascades in power grids. Science 2017, 358, eaan3184. [CrossRef] [PubMed]

4. Wang, A.S.; Luo, Y.; Tu, G.Y.; Liu, P. Vulnerability assessment scheme for power system transmission networks based on the fault chain theory. IEEE Trans. Power Syst. 2011, 26, 442-450. [CrossRef]

5. Gao, J.; Liu, X.; Li, D.; Havlin, S. Recent progress on the resilience of complex networks. Energies 2015, 8, 12187-12210. [CrossRef]

6. Zhu, Y.H.; Yan, J.; Sun, Y.; He, H.B. Revealing cascading failure vulnerability in power systems using risk-graph. IEEE Trans. Parallel Distrib. Syst. 2014, 25, 3274-3284. [CrossRef]

7. Fang, R.M.; Shang, R.Y.; Wang, Y.D.; Guo, X.H. Identification of vulnerable lines in power grids with wind power integration based on a weighted entropy analysis method. Int. J. Hydrog. Energy 2017, 42, 20269-20276. [CrossRef]

8. Kinney, R.; Crucitti, P.; Albert, R.; Latora, V. Modeling cascading failures in the North American power system. Eur. Phys. J. B Condens. Matter Phys. 2005, 46, 101-107. [CrossRef]

9. Dwivedi, A.; Yu, X. A maximum-flow-based complex network approach for power system vulnerability analysis. IEEE Trans. Ind. Inf. 2013, 9, 81-88. [CrossRef] 
10. Fang, J.K.; Su, C.; Chen, Z.; Sun, H.S.; Lund, P. Power system structural vulnerability assessment based on an improved maximum flow approach. IEEE Trans. Smart Grid 2016, 9, 777-785. [CrossRef]

11. Hao, B.; Miao, S.H. Hybrid flow betweenness approach for identification of vulnerable line in power system. IET Gener. Transm. Distrib. 2015, 9, 1324-1331. [CrossRef]

12. Gupta, S.; Kazi, F.; Wagh, S.; Singh, N. Analysis and prediction of vulnerability in smart power transmission system: A geometrical approach. Int. J. Electr. Power Energy Syst. 2018, 94, 77-87. [CrossRef]

13. Fan, W.L.; Zhang, X.M.; Mei, S.W.; Huang, S.W.; Wei, W.; Ding, L.J. Vulnerable transmission line identification using ISH theory in power systems. IET Gener. Transm. Distrib. 2018, 12, 1014-1020. [CrossRef]

14. Wang, Z.Q.; He, J.H.; Nechifor, A.; Zhang, D.H.; Crossley, P. Identification of Critical Transmission Lines in Complex Power Networks. Energies 2017, 10, 1294. [CrossRef]

15. Li, R.; Lv, Z.Y.; Zhai, C.X.; Li, T.B. The comprehensive identification model of critical lines in electric power system based on utility theory and cooperative game. In Proceedings of the 2016 IEEE PES Transmission \& Distribution Conference and Exposition-Latin America, Morelia, Mexico, 20-24 September 2016.

16. Liu, Y.; Singh, C. A methodology for evaluation of hurricane impact on composite power system reliability. IEEE Trans. Power Syst. 2011, 26, 145-152. [CrossRef]

17. Batts, M.E.; Simiu, E.; Russell, L.R. Hurricane wind speeds in the United States. J. Struct. Div. 1980, 106, 2001-2016.

18. Ministry of Housing and Urban-Rural Construction of the People's Republic of China. Load Code for the Design of Building Structure; GB50009-2012; China Architecture \& Building Press: Beijing, China, 2012.

19. Chen, Y.; Wang, S.Y.; Chen, B.; Xu, T.S.; Chen, Y.; Yu, J.L. Evaluation of the failure probability of power transmission corridors during typhoons using digital elevation information. Power Syst. Technol. 2017. [CrossRef]

20. Lin, Z.Z.; Wen, F.S.; Wang, H.F.; Lin, G.Q.; Mo, T.W.; Ye, X.J. CRITIC-based node importance evaluation in skeleton-network reconfiguration of power grids. IEEE Trans. Circuits Syst. II Express Briefs 2018, 65, $206-210$. [CrossRef]

21. Lin, Z.Z.; Wen, F.S.; Xue, Y.S. A restorative self-healing algorithm for transmission systems based on complex network theory. IEEE Trans. Smart Grid 2017, 7, 2154-2162. [CrossRef]

22. Guo, S.H.; Lu, Z.M. The Basic Theory of Complex Network; Science Press: Beijing, China, 2012.

23. Bialek, J. Tracing the flow of electricity. IEE Proc. Gener. Transm. Distrib. 1996, 143, 313-320. [CrossRef]

24. Ni, X.P.; Mei, S.W.; Zhang, X.P. Transmission lines vulnerability assessment based on complex network theory. Autom. Electr. Power Syst. 2008, 32, 1-5. [CrossRef]

25. Mirjat, N.H.; Uqaili, M.A.; Harijan, K.; Mustafa, M.W.; Rahman, M.M.; Khan, M.W. Multi-criteria analysis of electricity generation scenarios for sustainable energy planning in Pakistan. Energies 2018, 11, 757. [CrossRef]

26. Lu, J.; Wang, Y. A safety assessment of china's crude oil import based on G1 method. Procedia Soc. Behav. Sci. 2013, 96, 1738-1744. [CrossRef]

27. Lin, Z.Z.; Wen, F.S.; Huang, J.S. Evaluation of black-Start schemes employing entropy weight-based decision-making theory. J. Energy Eng. 2010, 136, 42-49. [CrossRef]

28. Wang, Y.J. A fuzzy multi-criteria decision-making model based on simple additive weighting method and relative preference relation. Appl. Soft Comput. 2015, 30, 412-420. [CrossRef]

29. He, L.; Li, C.L.; Nie, Q.Y.; Men, Y.; Zhu, J. Core abilities evaluation index system exploration and empirical study on distributed PV-generation projects. Energies 2017, 10, 2083. [CrossRef]

30. Zhao, H.; Li, N.N. Optimal siting of charging stations for electric vehicles based on fuzzy Delphi and hybrid multi-criteria decision making approaches from an extended sustainability perspective. Energies 2016, 9, 270. [CrossRef]

31. Dong, J.; Li, R.; Huang, H. Performance evaluation of residential demand response based on a modified fuzzy VIKOR and scalable computing method. Energies 2018, 11, 1097. [CrossRef]

(C) 2018 by the authors. Licensee MDPI, Basel, Switzerland. This article is an open access article distributed under the terms and conditions of the Creative Commons Attribution (CC BY) license (http://creativecommons.org/licenses/by/4.0/). 